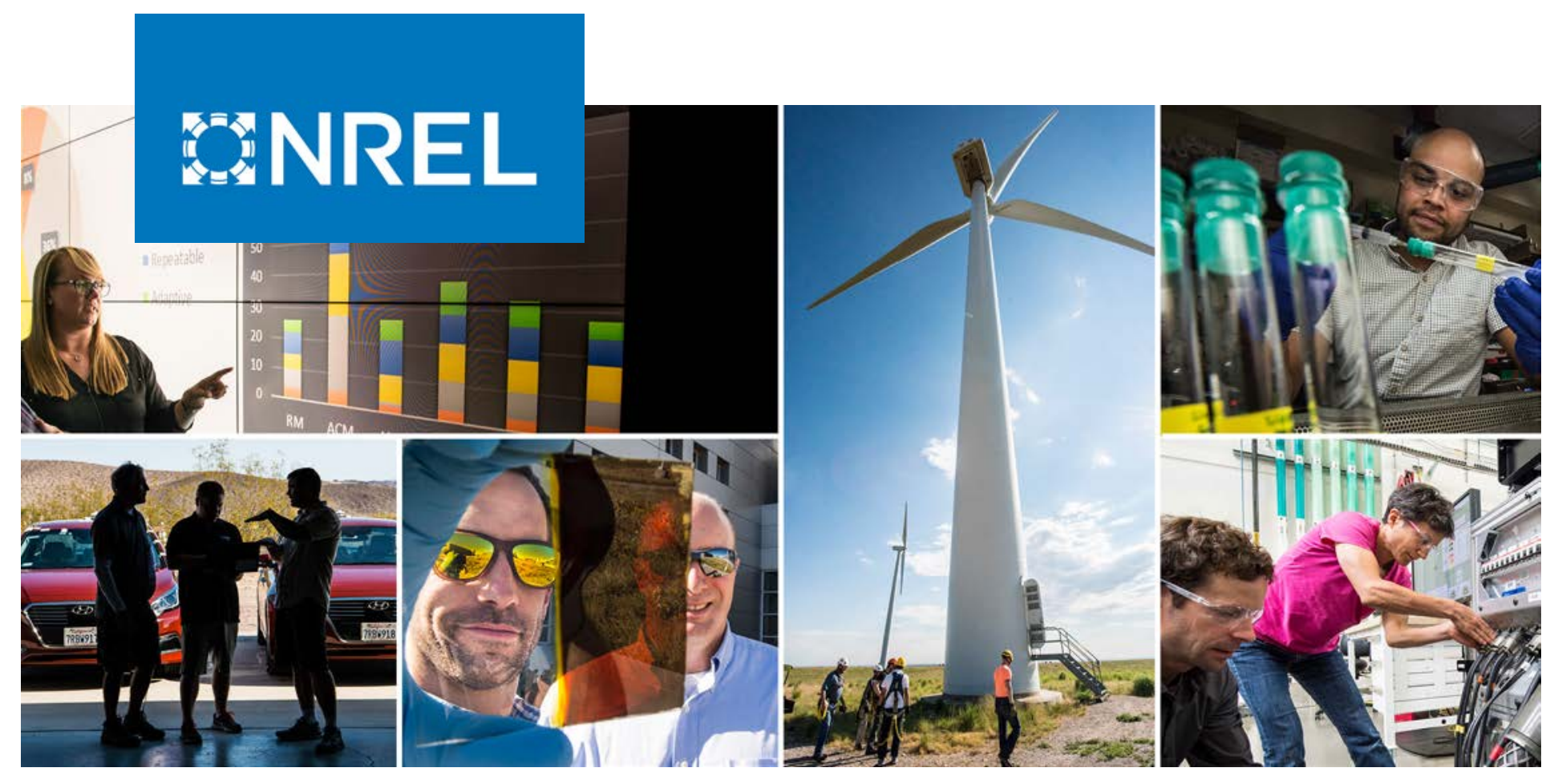

\title{
ADP-Based Home Energy Management System: A Case Study Using Dynamo
}

\section{Preprint}

Mohammad Faqiry, ${ }^{1}$ Li Wang, ${ }^{1}$ Hongyu Wu, ${ }^{1}$ Dheepak Krishnamurthy, ${ }^{2}$ and Bryan Palmintier ${ }^{2}$

${ }^{1}$ Kansas State University

${ }^{2}$ National Renewable Energy Laboratory

Presented at the Power and Energy Society (PES) General Meeting Portland, Oregon

August 5-10, 2018

NREL is a national laboratory of the U.S. Department of Energy

Office of Energy Efficiency \& Renewable Energy

Operated by the Alliance for Sustainable Energy, LLC

This report is available at no cost from the National Renewable Energy Laboratory (NREL) at www.nrel.gov/publications.
Conference Paper

NREL/CP-5D00-70422

June 2019 


\title{
GNREL
}

\section{ADP-Based Home Energy Management System: A Case Study Using Dynamo}

\section{Preprint}

\author{
Mohammad Faqiry, ${ }^{1}$ Li Wang, ${ }^{1}$ Hongyu Wu, ${ }^{1}$ \\ Dheepak Krishnamurthy, ${ }^{2}$ and Bryan Palmintier ${ }^{2}$ \\ ${ }^{1}$ Kansas State University \\ ${ }^{2}$ National Renewable Energy Laboratory
}

\section{Suggested Citation}

Faqiry, Mohammad, Li Wang, Hongyu Wu, Dheepak Krishnamurthy, and Bryan

Palmintier. 2019. ADP-Based Home Energy Management System: A Case Study Using

Dynamo: Preprint. Golden, CO: National Renewable Energy Laboratory.

NREL/CP-5D00-70422. https://www.nrel.gov/docs/fy19osti/70422.pdf.

NREL is a national laboratory of the U.S. Department of Energy Office of Energy Efficiency \& Renewable Energy Operated by the Alliance for Sustainable Energy, LLC

This report is available at no cost from the National Renewable Energy Laboratory (NREL) at www.nrel.gov/publications.

Contract No. DE-AC36-08GO28308
Conference Paper NREL/CP-5D00-70422 June 2019

National Renewable Energy Laboratory 15013 Denver West Parkway Golden, CO 80401

303-275-3000 • www.nrel.gov 


\section{NOTICE}

This work was authored by the National Renewable Energy Laboratory, operated by Alliance for Sustainable Energy, LLC, for the U.S. Department of Energy (DOE) under Contract No. DE-AC36-08GO28308. This work was supported by the Laboratory Directed Research and Development (LDRD) Program at NREL.. The views expressed herein do not necessarily represent the views of the DOE or the U.S. Government.

This report is available at no cost from the National Renewable Energy Laboratory (NREL) at www.nrel.gov/publications.

U.S. Department of Energy (DOE) reports produced after 1991 and a growing number of pre-1991 documents are available free via www.OSTI.gov.

Cover Photos by Dennis Schroeder: (clockwise, left to right) NREL 51934, NREL 45897, NREL 42160, NREL 45891, NREL 48097, NREL 46526.

NREL prints on paper that contains recycled content. 


\section{ADP-based Home Energy Management System: A Case Study using DYNAMO}

\author{
M.N. Faqiry, L. Wang, H. Wu \\ Department of Electrical \& Computer Engineering \\ Kansas State University, Manhattan KS, 66506
}

\begin{abstract}
This paper explores Home Energy Management System (HEMS) algorithms to minimize household cost while maintaining comfort when faced with uncertain weather, and demand. Specifically, we consider a HEMS that optimizes forward looking schedules for a home's heating, ventilation, and air conditioning (HVAC); water heater (WH); and electric vehicle (EV) charging while considering uncertainty in outside temperature, hot water usage, and non-controllable load (NCL). We adopt a Dynamic Programming (DP) formulation and utilize the Dynamic programming for Adaptive Modeling and Optimization (DYNAMO) toolkit to implement DP and approximate dynamic programming (ADP) algorithms. Simulation results under a single tariff plan compare the quality of the solution generated by ADP to that of DP, and show significant improvement in computation time while maintaining acceptable solution accuracy.
\end{abstract}

Index Terms-Approximate dynamic programming, demand response, electric vehicle, home energy management system, stochastic mixed-integer programming

\section{NOMENCLATURE}

$\mathcal{D} \quad$ Discomfort Function

C Cost Function

$s_{t}^{\text {HVAC }}$ State of HVAC at time $\mathrm{t}\left({ }^{\circ} \mathrm{F}\right)$

$s_{t}^{\mathrm{WH}} \quad$ State of $\mathrm{WH}$ at time $\mathrm{t}\left({ }^{\circ} \mathrm{F}\right)$

$s_{t}^{\mathrm{EV}} \quad$ State of EV at time $\mathrm{t}(\%$ State of Charge (SOC))

$x_{t}^{\text {HVAC }}$ Decision for HVAC at time $\left(^{\circ} \mathrm{F}\right)$

$x_{t}^{\mathrm{WH}} \quad$ Decision for $\mathrm{WH}$ at time $\mathrm{t}\left({ }^{\circ} \mathrm{F}\right)$

$x_{t}^{\mathrm{EV}} \quad$ Decision for EV at time t (\% SOC)

$\widetilde{u}_{t}^{\text {OUT }}$ Stochastic outside temperature ( $\left.{ }^{\circ} \mathrm{F}\right)$

$\tilde{u}_{t}^{\mathrm{WH}} \quad$ Stochastic hot water demand of household (gal./hr)

$\widetilde{u}_{t}^{\mathrm{NCL}} \quad$ Stochastic NCL demand of household (kW)

$\alpha_{t}^{\mathcal{D}} \quad$ Weighted sum coefficient for discomfort

$\alpha_{t}^{\mathcal{C}} \quad$ Weighted sum coefficient for cost

$\mathrm{s}_{t}^{\text {HVACd }}$ Desired state of HVAC at time $\mathrm{t}\left({ }^{\circ} \mathrm{F}\right)$

$\mathrm{s}_{t}^{\mathrm{WHd}}$ Desired state of $\mathrm{WH}$ at time $\mathrm{t}\left({ }^{\circ} \mathrm{F}\right)$

$s_{t_{d}}^{\text {EVd }}$ Desired state of EV at departure time $t_{d}(\%$ SOC)

$s_{\mathrm{t}_{\mathrm{d}}}^{\mathrm{EVd}} \quad$ State of EV at departure time $\mathrm{t}_{\mathrm{d}}(\% \mathrm{SOC})$

$\beta_{t}^{\mathrm{HVAC}}$ HVAC discomfort coefficient

$\beta_{t}^{\mathrm{WH}} \quad$ WH discomfort coefficient

$\beta_{t_{d}}^{E V} \quad$ EV discomfort coefficient at departure time $t_{d}$

$c_{t} \quad$ Price of electricity at time $\mathrm{t}(\$ / \mathrm{kWhr})$

This work was supported by the U.S. Department of Energy under Contract No. DE-AC36-08-GO28308 with Alliance for Sustainable Energy, the Manager and Operator of the National Renewable Energy Laboratory (NREL) though the Laboratory Directed Research and Development Program. The U.S. Government retains and the publisher, by accepting the article for publication, acknowledges that the U.S. Government retains a nonexclusive, paid-up, irrevocable, worldwide license to publish or reproduce the published form of this work, or allow others to do so, for U.S. Government purposes.

\author{
D. Krishnamurthy, and B. Palmintier \\ Power Systems Engineering Center, National Renewable \\ Energy Laboratory, Golden CO, 80401
}

$p_{t}^{G} \quad$ Power purchased from the grid (kW)

$\gamma^{\mathrm{RM}} \quad$ Room insulation thermal efficiency coefficient

$\gamma^{\text {OUT }}$ Outside temperature thermal influence coefficient

$\gamma^{\mathrm{HVAC}}$ HVAC unit conversion coefficient $\left(\mathrm{kW} /{ }^{\circ} \mathrm{F}\right)$

$\underline{\mathrm{S}}_{t}^{\mathrm{HVAC}}$ Min. temperature setting for HVAC at time $\mathrm{t}\left({ }^{\circ} \mathrm{F}\right)$

$\bar{s}_{t}^{\mathrm{HVAC}}$ Max. temperature setting for HVAC at time $\mathrm{t}\left({ }^{\circ} \mathrm{F}\right)$

$\overline{\mathrm{x}}^{\mathrm{HVAC}}$ Max. HVAC decision limit $\left({ }^{\circ} \mathrm{F}\right)$

$\underline{x}^{\mathrm{HVAC}}$ Min. HVAC decision limit $\left({ }^{\circ} \mathrm{F}\right)$

$\gamma^{\mathrm{WH}, \mathrm{C}}$ Thermal natural cooling coefficient of WH

$\gamma^{\mathrm{USG}} \quad$ Hot water usage unit conversion coefficient $\left({ }^{\circ} \mathrm{F} / \mathrm{gal}\right)$

$\gamma^{\mathrm{WH}} \quad \mathrm{WH}$ unit conversion coefficient $\left(\mathrm{kW} /{ }^{\circ} \mathrm{F}\right)$

$\underline{\mathrm{s}}_{t}^{\mathrm{WH}} \quad$ Min. temp. setting for $\mathrm{WH}$ at time $\mathrm{t}\left({ }^{\circ} \mathrm{F}\right)$

$\overline{\mathrm{s}}_{t}^{\mathrm{WH}} \quad$ Max. temp. setting for $\mathrm{WH}$ at time $\mathrm{t}\left({ }^{\circ} \mathrm{F}\right)$

$\underline{x}_{t}^{\text {WH }} \quad$ Min. WH decision limit $\left({ }^{\circ} \mathrm{F}\right)$

$\overline{\mathrm{x}}_{t}^{\mathrm{WH}}$ Max. WH decision $\left({ }^{\circ} \mathrm{F}\right)$

$\gamma^{\mathrm{EV}} \quad \mathrm{EV}$ unit conversion coefficient $(\mathrm{kW} / \% \mathrm{SOC})$

$\gamma^{\mathrm{EV}, \mathrm{L}} \quad$ EV energy loss (self-discharge) coefficient

$\gamma^{\mathrm{EV}, \mathrm{C}} \quad$ EV charging efficiency coefficient

$\gamma^{\mathrm{EV}, \mathrm{NCL}} \mathrm{EV}$ SOC unit conversion coefficient (\% SOC/kWh)

$\underline{\mathbf{s}}_{t}^{\mathrm{EV}} \quad$ EV minimum state of charge

$\bar{s}_{t}^{\mathrm{EV}} \quad$ EV maximum state of charge

$\underline{\mathrm{x}}_{t}^{\mathrm{EV}} \quad \mathrm{EV}$ minimum decision limit (\% SOC)

$\overline{\mathrm{x}}_{t}^{\mathrm{EV}} \quad \mathrm{EV}$ maximum decision limit (\% SOC)

$t_{a} / t_{d} \quad$ Arrival/departure time of EV

$\Delta t \quad$ Time interval resolution

$\mathrm{T} \quad$ Look-ahead horizon

\section{INTRODUCTION}

Automated home energy management systems (HEMS) can help residential energy users realize the full potential of demand response (DR) schemes by optimally scheduling their distributed energy resources (DER) [1]. The goal of HEMS is to provide systems that provide autonomous control of appliances to limit the need for user intervention while minimizing cost and maximizing comfort [2]. Market analysis studies indicate that there is a growing consumer awareness of the potential of HEMS to help manage home energy consumption and energy costs [3]. This presents an opportunity to develop efficient and computationally tractable algorithms that can be embedded in HEMS products. In this context, stochastic variables such as electricity prices, weather conditions, and user consumption behavior introduce 
challenges when attempting to optimize the schedules of home appliances and other energy uses.

A stochastic dynamic programing (DP) formulation can capture this complexity and has been used successfully for HEMS in the past [16]. However, traditional DP faces multiple curses of dimensionality that limit scalability [4]. Approximate dynamic programming (ADP) is a large class of algorithms that use various approaches to speed up DP and enable its use for larger optimal decision making under uncertainty [4], [5].

A number of past HEMS efforts have explored using ADP for PV-battery management [1], [6]-[8]. In [1], authors use ADP with temporal difference learning to implement a computationally efficient HEMS that optimizes PV-battery system scheduling for minimal cost and user discomfort. Reference [6] proposes a novel distributed iterative ADP to solve the multi-battery optimal coordination control problems. Reference [7] develops a self-learning scheme based on ADP to optimize residential energy systems. Reference [8] compares different methods for home energy resource scheduling and analyzes their performance dependency on the battery model employed, focusing on battery capacity and charge/discharge rates. A few other studies have used ADP to control energy use of building cooling systems to minimize energy consumption, while preserving occupant comfort [9], [10].

In this paper, we focus on scheduling multiple appliances and use HEMS to determine setpoints for the heating, ventilation, and air conditioning (HVAC) system; setpoints for the water heater $(\mathrm{WH})$, and the charge/discharge schedule for an electric vehicle (EV). The HEMS minimizes expected cost and discomfort to the household, under a range of uncertainties. To the best of our knowledge, this is the first of its kind to model and optimize multiple appliances schedules using ADP. We use the DYNAMO toolkit developed by the National Renewable Energy Laboratory (NREL) to easily implement the DP and ADP algorithms and enable easy and fair comparisons.

\section{MODEL}

Consider the HEMS schematic in Fig.1. The goal is to minimize a weighted sum of the household's discomfort and cost by determining the HVAC setting, WH setting, and EV charge/discharge schedule "here and now" while looking forward multiple time steps in the future to also endogenously capture future uncertainty. This problem can be modeled in terms of a DP with states, decisions, and stochastic variables. At every time step, the HVAC, WH, and EV states are respectively considered as the room temperature, water temperature, and EV battery state-of-charge (SOC). The decision set captures whether or not to run the HVAC system and at what fan speed, whether or not to heat water in the $\mathrm{WH}$, and how much energy to inject into the EV battery. We also consider uncertainty in outside temperature, hot water demand, and other non-controllable loads (NCL) of the household. In addition, we make the following timing assumptions:

1) The appliance is in state $s_{t}$ when HEMS makes a decision $x_{t}$ at the beginning of the time step.

2) The decision is applied immediately and impacts the remainder of the time step.

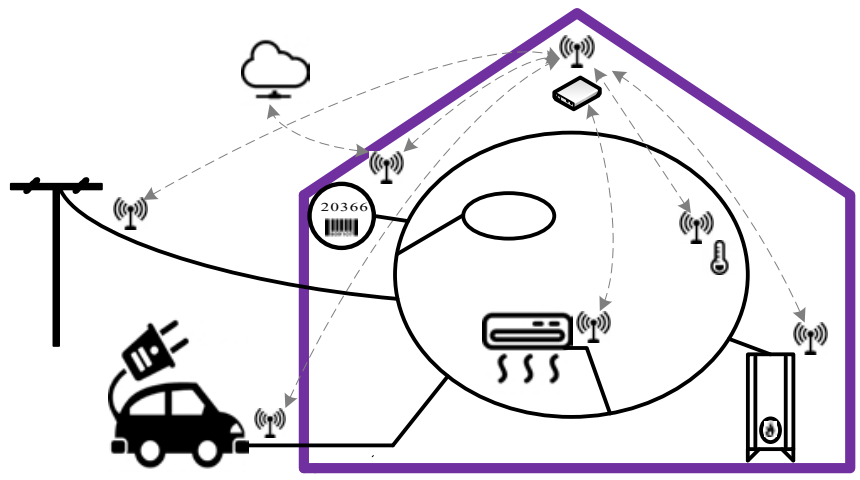

Figure 1. Home Energy Management System schematic

3) The stochastic variable associated with the appliance is realized after the decision is applied and lasts for the remainder of the time step.

As an example, Fig. 2 diagrams the HVAC cooling setting portion of problem as a decision tree. Here, squares represent decision nodes, circles represent uncertainty nodes, and

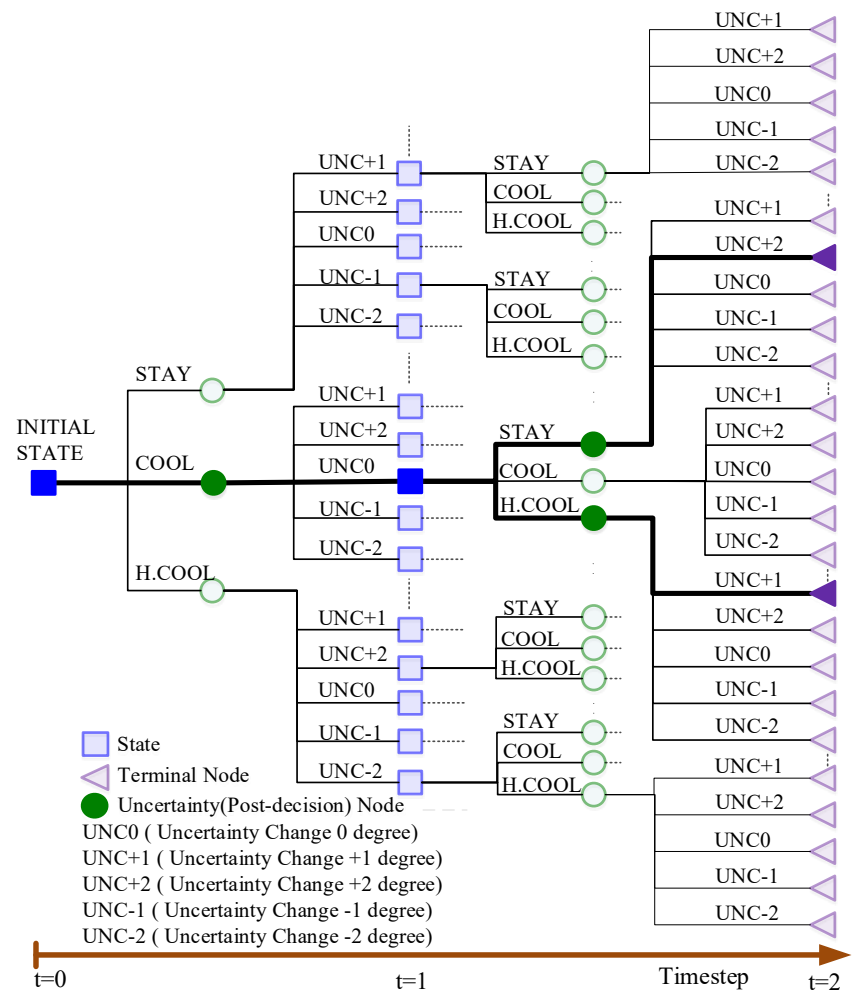

Figure 2. Decision tree for HVAC with highlighted route that minimizes expected weighted sum of cost and discomfort.

triangles show terminal nodes. The decisions are STAY (no action), COOL, or H.COOL (highly cool). The outside temperature is drawn from historic data, but its forecast is estimated as a generalized random walk that may go up or down a few degrees or remain unchanged. The optimal DP solution can be found using backward induction [11] as follows: 
1. Compute the thermal discomfort at the terminal nodes.

2. Compute the expected cost and discomfort of each preceding uncertainty node.

3. For each decision node, pick the decision with lowest sum of weighted decision cost (energy and discomfort) and expected future value.

4. Repeat steps 2 and 3, working backward in time, until the optimal first period decision is found.

Mathematically, the state, decision, and uncertainty sets tuple $\left\{\boldsymbol{s}_{t}, \boldsymbol{x}_{t}, \widetilde{\boldsymbol{u}}_{t}\right\}$ covers HVAC, WH and EV for all time periods, i.e. $\forall \mathrm{t} \in\{1, \ldots, \mathrm{T}\}$,

$$
\left\{\boldsymbol{s}_{t}, \boldsymbol{x}_{t}, \tilde{\boldsymbol{u}}_{t}\right\}=\left\{\begin{array}{c}
\left(s_{t}^{\mathrm{HVAC}}, s_{t}^{\mathrm{WH}}, s_{t}^{\mathrm{EV}}\right),\left(x_{t}^{\mathrm{HVAC}}, x_{t}^{\mathrm{WH}}, x_{t}^{\mathrm{EV}}\right), \\
\left(\tilde{u}_{t}^{\mathrm{OUT}}, \tilde{u}_{t}^{\mathrm{WH}}, \tilde{u}_{t}^{\mathrm{NCL}}\right)
\end{array}\right\} .
$$

The HEMS objective can be achieved in terms of the following optimization problem using a form of Bellman's equation [5], [12].

$$
\begin{gathered}
\text { Minimize with respect to } x_{t}^{\mathrm{HVAC}}, x_{t}^{\mathrm{WH}}, x_{t}^{\mathrm{EV}}: \\
\mathbb{E}\left\{\sum_{t=0}^{T} \alpha_{t}^{\mathcal{D}} \cdot \mathcal{D}_{t}\left(\boldsymbol{s}_{t}, \boldsymbol{x}_{t}, \widetilde{\boldsymbol{u}}_{t}\right)+\alpha_{t}^{\mathcal{C}} \cdot \mathcal{C}_{t}\left(\boldsymbol{s}_{t}, \boldsymbol{x}_{t}, \tilde{\boldsymbol{u}}_{t}\right)\right\}
\end{gathered}
$$

Subject to:

$$
\text { HVAC, WH, EV constraints } \forall \mathrm{t} \in\{1, \ldots, \mathrm{T}\}
$$

The objective function in (1) shows the expected weighted sum of discomfort $\mathcal{D}_{t}$ and $\operatorname{cost} \mathcal{C}_{t}$ over the look-ahead horizon. The discomfort and cost functions are given below.

$$
\left.\begin{array}{l}
\mathcal{D}_{t}\left(\boldsymbol{s}_{t}, \boldsymbol{x}_{t}, \tilde{\boldsymbol{u}}_{t}\right)=\sum_{t=1}^{T}\left(\begin{array}{c}
\beta_{t}^{\mathrm{HVAC}}\left(\mathrm{s}_{t}^{\mathrm{HVACd}}-s_{t}^{\mathrm{HVAC}}\right)^{2} \\
+\beta_{t}^{\mathrm{WH}}\left(\mathrm{s}_{t}^{\mathrm{WHd}}-s_{t}^{\mathrm{WH}}\right)^{2}
\end{array}\right) \\
+\beta_{\mathrm{t}_{\mathrm{d}}}^{\mathrm{EV}}\left(\mathrm{s}_{\left.\mathrm{t}_{\mathrm{d}}^{\mathrm{EVd}}-s_{\mathrm{t}_{\mathrm{d}}}^{\mathrm{EVd}}\right)^{2}}\right.
\end{array}\right)
$$

In (2), the discomfort due to each appliance is modeled as the square of the deviation from their respective desired values. Each discomfort term is weighted differently to accommodate the user's preferences for each appliance. For EV, only the departure time dissatisfaction is included. This is because we assume the user only cares about the departure time SOC and may not experience any discomfort due to low SOC when the $\mathrm{EV}$ is at home. The cost function in (3) is computed as the total electricity cost of the imported grid power over the look ahead horizon. Here, every household is assumed to be a price taker. The imported grid power is computed as the sum of decisions, i.e. $p_{t}^{\mathrm{G}}=\gamma^{\mathrm{HVAC}} x_{t}^{\mathrm{HVAC}}+\gamma^{\mathrm{WH}} x_{t}^{\mathrm{WH}}+\gamma^{\mathrm{EV}} x_{t}^{\mathrm{EV}}$.

The constraints in (1) are given as the state transition functions and other limiting constraints explained below.

HVAC:

$$
s_{t}^{\mathrm{HVAC}}=\left\{\begin{array}{l}
\gamma^{\mathrm{RM}} s_{t-1}^{\mathrm{HVAC}}-x_{t}^{\mathrm{HVAC}}+\gamma^{\mathrm{OUT}} \tilde{u}_{t}^{\mathrm{OUT}}, \text { if cooling } \\
\gamma^{\mathrm{RM}} s_{t-1}^{\mathrm{HVAC}}+x_{t}^{\mathrm{HVAC}}+\gamma^{\mathrm{OUT}} \tilde{u}_{t}^{\mathrm{OUT}}, \text { if heating }
\end{array}\right.
$$

$$
\begin{aligned}
& \underline{\mathrm{s}}_{t}^{\mathrm{HVAC}} \leq s_{t}^{\mathrm{HVAC}} \leq \overline{\mathrm{s}}_{t}^{\mathrm{HVAC}} \\
& \underline{\mathrm{x}}_{t}^{\mathrm{HVAC}} \leq x_{t}^{\mathrm{HVAC}} \leq \overline{\mathrm{x}}_{t}^{\mathrm{HVAC}} \\
& \underline{\mathrm{x}}_{t}^{\mathrm{HVAC}} \leq x_{t}^{\mathrm{HVAC}} \leq \overline{\mathrm{x}}_{t}^{\mathrm{HVAC}}
\end{aligned}
$$

Equation (4) shows the state transition of the room temperature for both cooling and heating modes that are predetermined. The room temperature is changed by the HVAC decision and the uncertain change is due to outside temperature. Note that in this control implementation, the HVAC efficiency is embedded in the cost function and building thermal properties as captured by building and outside air temperature driven factors $\gamma^{\mathrm{RM}}$ and $\gamma^{\mathrm{OUT}}$, respectively. Also, the native control decision, $x_{t}^{\mathrm{HVAC}}$, is computed in units of thermal energy added or removed. This control can be later adapted to corresponding thermostat settings. Equations (5)-(7) indicate that, for both cooling and heating cases, the states and the decisions have to lie within the specified bounds.

WH:

$$
\begin{aligned}
& s_{t}^{\mathrm{WH}}=\gamma^{\mathrm{WH}, \mathrm{C}} s_{t-1}^{\mathrm{WH}}+x_{t}^{\mathrm{WH}}-\gamma^{\mathrm{USG}} \tilde{u}_{t}^{\mathrm{WH}} \Delta t \\
& \underline{s}_{t}^{\mathrm{WH}} \leq s_{t}^{\mathrm{WH}} \leq \overline{\mathrm{s}}_{t}^{\mathrm{WH}} \\
& \underline{\mathrm{x}}_{t}^{\mathrm{WH}} \leq x_{t}^{\mathrm{WH}} \leq \overline{\mathrm{x}}_{t}^{\mathrm{WH}}
\end{aligned}
$$

Similarly, (8)-(10) show the water temperature state transition and the state and decision limit constraints of the $\mathrm{WH}$.

EV:

$$
s_{t}^{\mathrm{EV}}=\left\{\begin{array}{lr}
\gamma^{\mathrm{EV}, \mathrm{L}} s_{t-1}^{\mathrm{EV}}+\gamma^{\mathrm{EV}, \mathrm{C}} x_{t}^{\mathrm{EV}}-\gamma^{\mathrm{EV}, \mathrm{NCL}} \widetilde{u}_{t}^{\mathrm{NCL}} \Delta t, t_{a} \leq t \leq t_{d} \\
\text { Constant }, & \text { Otherwise }
\end{array}\right.
$$

$$
\begin{aligned}
& \underline{\mathrm{s}}_{t}^{\mathrm{EV}} \leq s_{t}^{\mathrm{EV}} \leq \overline{\mathrm{s}}_{t}^{\mathrm{EV}} \\
& -\underline{\mathrm{x}}_{t}^{\mathrm{EV}} \leq x_{t}^{\mathrm{EV}} \leq \overline{\mathrm{x}}_{t}^{\mathrm{EV}}
\end{aligned}
$$

When the EV is at home, (11) captures its SOC loss by selfdischarge over time [13], charging decision of HEMS, and the amount of random NCL consumed. In this paper, it is assumed that when EV is at home, it feeds the household stochastic NCL. However, when EV is not at home, the grid directly feeds the stochastic NCL, and HEMS only optimizes decisions for HVAC and WH. Equations (12) and (13) indicate that the EV state and decision must lie within the specified bounds.

\section{Simulation Results}

The HEMS model was coded in MATLAB using the standard DYNAMO toolkit problem structure, which enables solving with both DP and ADP without changes. We used the simple sampled backward induction ADP algorithm which works similarly to traditional DP, but only considers a sampled subset of states, decisions, and uncertainty realizations. These are used to build a function approximation - we selected a local average - of the post-decision value function that is used to inform the previous period decision.

The discomfort and cost function coefficients, $\alpha_{t}^{\mathcal{D}}$ and $\alpha_{t}^{\mathcal{C}}$ in the objective function were equally weighted. HVAC parameters in the state transition expression in (4) were 
obtained by applying linear regression to a historical data set for a residential house located in Hillsboro, Oregon [2], [14]. A cooling case is studied here. WH parameters were extracted from [15]. EV battery parameters and the forecast data for outside temperature, hot water demand, and NCL were adopted from [14]. For both DP and ADP simulations, appliances range of operation and other data are summarized in Table I.

TABLE I. SimULATION PARAMETERS For HVAC, WH, \& EV

\begin{tabular}{c|c|c}
\hline \multicolumn{3}{c}{ Appliance Range of Operation and other Parameters } \\
\hline Algorithm & DP Backward Induction & $\begin{array}{c}\text { ADP Sampled Backward } \\
\text { Induction }\end{array}$ \\
\hline State Range & $\begin{array}{c}\{[70,85],[108,118], \\
[20,100]\}\end{array}$ & $\begin{array}{c}\{[70,85],[108,118], \\
[20,100]\}\end{array}$ \\
\hline $\begin{array}{c}\text { Stochastic Variable } \\
\text { Range }\end{array}$ & $\{\{85,100\},\{0,10\},\{0,5\}\}$ & $\{\{85,100\},\{0,10\},\{0,12\}\}$ \\
\hline Desired Values & $\{77,113,92\}$ & $\{77,113,92\}$ \\
\hline $\begin{array}{c}\text { EV } \\
\text { Arrival/Departure } \\
\text { Time }\end{array}$ & $\{17,9\}$ & $\{17,9\}$ \\
\hline State Samples & 3600 ( Total \#of States) & 1200 \\
\hline Decision Samples & NA & 60 \\
\hline Uncertainty Samples & NA & 30 \\
\hline
\end{tabular}

We adopt the Pacific Gas \& Electric (PG\&E) time-of-use electricity rate, ETOU-E6 (Fig. 3). This plan contains three price levels: $\$ 0.24 / \mathrm{kWh}, \$ 0.32 / \mathrm{kWh}$, and $\$ 0.44 / \mathrm{kWh}$ that are referred to as base, Peak A, and Peak B prices. Peak A is applied during hours 10-12, 19, and 20, and Peak B is applied during hours 13-18. In the remaining hours, base prices are applied. Since both DP and ADP compute the current decision by looking forward, two separate cases were simulated as follows.

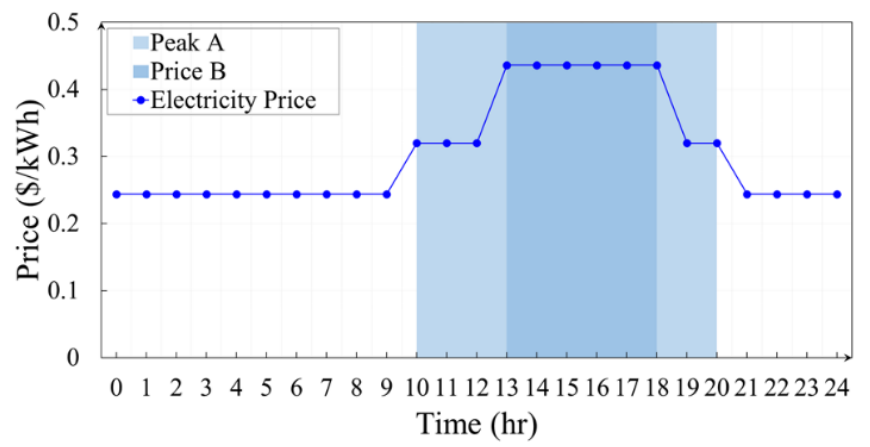

Figure 3. The PG\&E ETOU-B time of use electricity tariff.

The first case is simulated to show the performance of ADP versus DP. In this case, both DP and ADP were run only once to optimize for the current decision by looking ahead for 24 hours. Fig. 4 shows the expected household utility generated by DP and, with different state samplings, for ADP. Notice that increasing the number of state samples returns a higher expected utility. With 1200 or more state samples, ADP returns a good enough solution compared to DP while performing much faster. Table II shows computation time of ADP compared to DP for varying state sample sizes. Increasing the number of state samples increases the ADP computation time sublinearly, while the solution converges to that from DP, rapidly at first then roughly asymptotically. However, even at the maximum considered number of 1200 samples, ADP still computes the decision in 508s while DP takes nearly ten times longer at $5011 \mathrm{~s}$. The DP solution takes longer as it exhaustively traverses the entire state, decision, and uncertainty spaces to find the optimal decision.

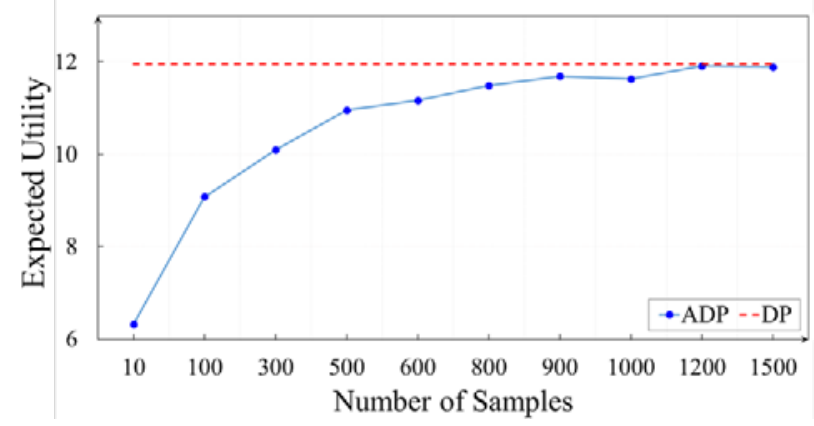

Figure 4. Household expected utility, i.e. objective function, attained by ADP with increasing number of samples.

TABLE II. ADP \& DP RUn Time For Different State SAMPLE Sizes

\begin{tabular}{c|c|ccccccccc}
\hline DP & Time (s) & 5011 & & & & & & & & \\
\hline \multirow{2}{*}{ ADP } & Samples \# & 50 & 100 & 300 & 500 & 600 & 800 & 900 & 1000 & 1200 \\
\cline { 2 - 11 } & Time (s) & 66 & 103 & 205 & 277 & 314 & 382 & 415 & 442 & 508 \\
\hline
\end{tabular}

The second case was simulated to examine the HEMS decisions for 24 hours starting at 12:00 am. At every hour, ADP with 1200 samples and 12-hour look-ahead was implemented in a rolling window fashion to generate $\mathrm{HVAC}, \mathrm{WH}$, and $\mathrm{EV}$ decisions for the current time step. The results of this simulation are shown in Fig. 5 along with shaded regions showing Peak A and B prices. In each figure, the horizontal dotted line shows the target state and the line graph (both solid and dotted) with square and circle markers show the HEMS-estimated state transitions as a result of the HEMS decision and stochastic variable impact. The bars at every hour show the first hour forecast of the 12-hour look ahead stochastic variable plus a small noise generated at random. During each hour, there are two data points shown by a square and a circle marker representing pre-decision and post-decision state respectively while matching Fig. 2. Each pre-decision state is connected to the post-decision state by a solid line representing decision impact and each post-decision state is connected to the next predecision state by a dotted line representing stochastic variable impact on the HEMS state.

Fig. 5 (a) shows the HEMS air temperature state for the case of cooling. Notice that the air temperature state fluctuates around the desired values of $77^{\circ} \mathrm{F}$ to minimize discomfort. There are multiple time steps at which HEMS precools the air temperature. At hour 9, when price is at baseline, HEMS initiates precooling before prices increase to Peak A and the outside temperature gets hotter. A similar precooling effect is observed for time step 12. Analogously, at hours 17 and 18, due to current high price and upcoming lower prices, HEMS does not take any cooling action and let the temperature warm up to minimize electricity cost. As soon as the price drops to Peak A level, the HEMS opts to cool the air considerably to minimize discomfort after successive hours with no cooling actions during higher prices. The effect of stochastic variable realization is clearly seen after each decision is applied.

Similar observations can be made for WH schedules shown in Fig. 5 (b). At hour 9, HEMS decides to preheat the water temperature significantly in preparation for expected high 
demand and price in upcoming hours. During hours with no water demand, the HEMS decides not to heat the water.

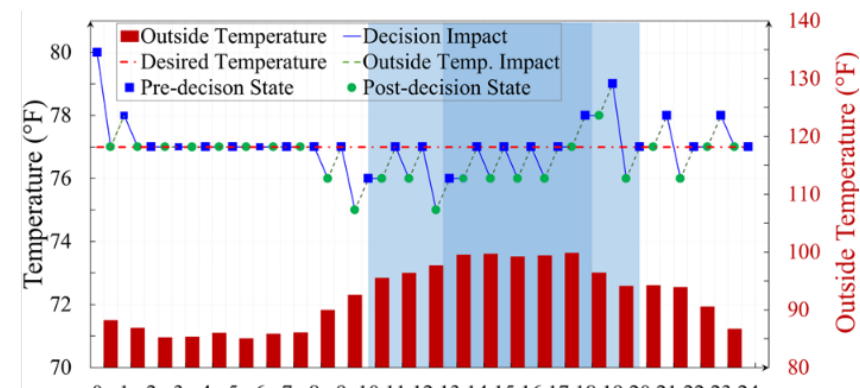

$\begin{array}{llllllllllll}0 & 1 & 2 & 3 & 4 & 5 & 6 & 7 & 8 & 9 & 1011 & 12131415161718192021222324\end{array}$ Time (hr)
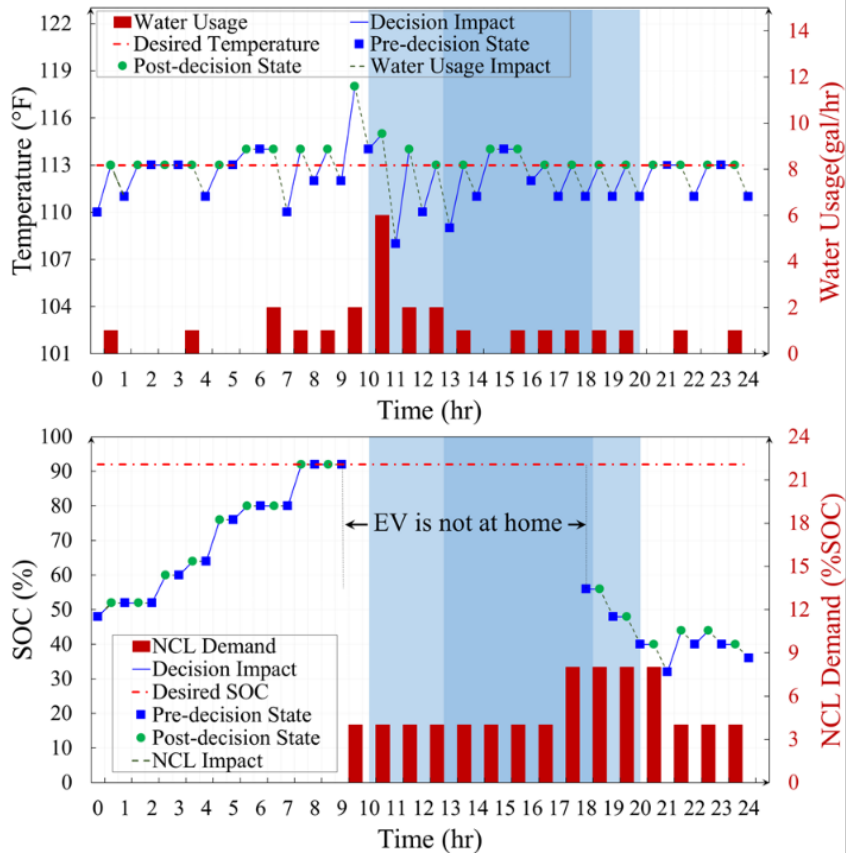

Figure 5. HEMS states, decisions, and uncertainty for 24 hours with 12 hour look-ahead (a) HVAV, (b) WH, and (c) EV.

For the EV, we assume that the initial SOC is at $48 \%$ and it departs at 9 am and arrives back at $6 \mathrm{pm}$ while travelling a known commuting distance that reduces the SOC by $36 \%$. Fig.5(c) shows that the deviation from the desired SOC is high during hours other than the departure time. This is because the discomfort due to EV SOC is applied only at the departure time, i.e., no penalty is applied for deviation from the desired SOC when EV is parked at home. Notice that initially, during certain hours, the HEMS decides to charge the EV to meet the desired SOC at the departure time. When driving, the SOC drops according to the driving distance and the efficiency of the EV battery, which is considered here to be $36 \%$. Since the EV arrives home during the Peak A prices, the HEMS postpones charging until the baseline price hours.

\section{CONCLUSIONS}

Based on numerical results, ADP offers a promising approach to HEMS-based appliance scheduling under uncertainty. In particular, careful selection of the number of state samples used in sampled backward induction can provide a very near optimal solution compared with DP in an order of magnitude less computation time. Further computational savings can be achieved with slight degradation in solution accuracy. We were able to rapidly develop the DP problem framing using DYNAMO and then seamlessly reuse the code to solve the problem in ADP.

Our future work will model ADP-based HEMS with more appliances such as dishwasher, washer/dryer, pool pumps, etc. and compare HEMS performance to other stochastic optimization approaches including commercial mixed integer linear programming (MILP) solvers [14]. We also plan to compare the controller estimates to more sophisticated physicsbased models for homes and end-uses. Looking ahead, DYNAMO also offers the opportunity to explore additional ADP algorithms and to apply similar approaches to other domains, such as distribution system planning and operation with stochastic distributed renewable generation.

\section{REFERENCES}

[1] C. Keerthisinghe, G. Verbič, and A. C. Chapman, "Energy management of PV-storage systems: ADP approach with temporal difference learning," 19th Power Syst. Comput. Conf. PSCC 2016, pp. 1-7, 2016.

[2] A. Pratt, B. Banerjee, and T. Nemarundwe, "Proof-of-concept home energy management system autonomously controlling space heating," IEEE Power Energy Soc. Gen. Meet., 2013.

[3] A. Pratt, D. Krishnamurthy, M. Ruth, H. Wu, M. Lunacek, and P. Vaynshenk, "Transactive Home Energy Management Systems: The Impact of Their Proliferation on the Electric Grid," IEEE Electrification Magazine 4, no. 4, pp. 8-14, 2016.

[4] W. B. Powell, Approximate Dynamic Programming: Solving the Curses of Dimensionality: Second Edition 2011.

[5] Bertsekas, "Approximate Dynamic Programming Lecture Notes," Dyn. Program. Optim. Control Athena Scientific, 2014.

[6] Q. Wei, D. Liu, G. Shi, and Y. Liu, "Multibattery Optimal Coordination Control for Home Energy Management Systems via Distributed Iterative Adaptive Dynamic Programming," IEEE Trans. on Ind. Electron., vol. 62, no. 7, pp. 4203-4214, 2015.

[7] T. Huang, and D. Liu. "Residential energy system control and management using adaptive dynamic programming." In IEEE International Joint Conference on Neural Networks (IJCNN), pp. 119124,2011

[8] S. Squartini, D. Fuselli, M. Boaro, F. De Angelis, and F. Piazza, "Home energy resource scheduling algorithms and their dependency on the battery model," IEEE Symp. on Comput. Intell. Appl. Smart Grid, pp. $122-129,2013$.

[9] F. Borghesan, R. Vignali, L. Piroddi, M. Prandini, and M. Strelec, "Approximate dynamic programming-based control of a building cooling system with thermal storage." In 4th IEEE/PES Innovative Smart Grid Technologies (ISGT) Europe, pp. 1-5, 2013.

[10] N. Ceriani, R. Vignali, L. Piroddi, and M. Prandini, "An approximate dynamic programming approach to the energy management of a building cooling system." In European Control Conference (ECC), pp. 20262031, 2013.

[11] B. Palmintier, D. Krishnamurthy, and Hongyu Wu, "Design flexibility for uncertain distributed generation from photovoltaics." In IEEE Power Energy Soc. Innov. Smart Grid Technol. Conf., pp. 1-5, 2016.

[12] R. Bellman, Dynamic Programming. Princeton University Press, Princeton, NJ, 1957

[13] K. C. Divya and J. Østergaard, "Battery energy storage technology for power systems-An overview," Electric Power System Research, vol. 79, no. 4, pp. 511-520, 2009.

[14] H. Wu, A. Pratt, and S. Chakraborty. "Stochastic optimal scheduling of residential appliances with renewable energy sources." In IEEE Power \& Energy Society General Meeting, pp. 1-5, 2015.

[15] J. L. Mathieu, "Modeling, Analysis, and Control of Demand Response Resources," Univ. Calif. Berkeley, PhD Diss., 2012.

[16] D. Livengood and R. C. Larson, "Energy Box: Locally Automated Optimal Control of Residential Electricity Usage," Service Science, vol. 1, pp. 1-16, 2009. 